\title{
Treatment Planning System (TPS) for Carbon lon Therapy: The INFN TPS project
}

\author{
Elke Schmitt* ${ }^{\dagger}$ \\ Experimental Physics Department, University of Turin \& Istituto Nazionale di Fisica Nucleare \\ (INFN) Torino \\ Via Pietro Giuria 1, 10125 Torino, Italy \\ E-mail: schmitteto.infn.it
}

\section{Andrea Attili}

INFN Torino

E-mail: attili@to.infn.it

\section{Flavio Marchetto}

INFN Torino

E-mail: fmarcheteto.infn.it

\section{Vincenzo Monaco}

Experimental Physics Department, University of Turin \& INFN Torino

E-mail: monaco@to.infn.it

\section{Cristiana Peroni}

Experimental Physics Department, University of Turin \& INFN Torino

E-mail: peroniato.infn.it

\section{Germano Russo}

Experimental Physics Department, University of Turin \& INFN Torino

E-mail: russo@to.infn.it

The Italian INFN TPS project is a multi-disciplinary effort by the Istituto Nazionale di Fisica Nucleare (INFN) to develop a fully functional Treatment Planning System (TPS) for therapies using carbon ion beams with an active delivery system. This paper presents general information about the project and gives an overview of two specific tasks of the INFN Torino, respectively the biological dose optimisation and the beam modelling. An example of the biological cost function and the related results for the optimised biological dose, obtained with the conjugate gradient method applied to a basic treatment case, are given. Moreover, it is shown how the radiobiological effectiveness can be modelled through a transfer function, derived from the physical measured data, when a ripple filter is inserted along the beam line. The work has been done in collaboration with the Belgian Ion Beam Applications (IBA) company, within a research cooperation agreement.

XLIX International Winter Meeting on Nuclear Physics

24-28 January 2011

BORMIO, Italy

\footnotetext{
*Speaker.

${ }^{\dagger}$ For the INFN TPS collaboration. This work was supported by University of Turin and partly by INFN \& IBA.
} 


\section{Introduction}

To start dealing with the problem of cancer, first some figures as a brief overview are given. Every year about 2.4 million new cancer cases appear in the EU and 1.2 million deaths are caused by cancer [1]. This makes cancer the second cause of death (25\%) directly after heart diseases (41\%) [2]. Worldwide there are about 7.6 million cancer deaths per year and it is the leading cause of deaths (about 13\%) [3].

The choice of the treatments depends in general on the type, location and size of the tumour. Radiotherapy is already now the second most commonly used cancer therapy method (about 50\%), directly after surgery and even before chemotherapy. In addition, the technical developments in radiotherapy and the growing knowledge in radiobiology are steadily increasing the applicability of this type of treatment.

\subsection{Hadrontherapy}

In modern cancer radiotherapy the use of charged particles, so far mainly protons and carbon ions, is favoured due to the following advantages over traditional X-rays radiotherapy:

- A better transverse and depth conformation which helps to spare the healthy tissue and the Organs At Risk (OAR) (fig. 1(a) and 1(b)).

- The possibility to adjust the depth of the maximum dose deposition by using different energies, which makes also possible the treatment of deep seated tumours (fig. 1(c)).

- A higher Relative Biological Effectiveness (RBE) for carbon ions, which enhances the ability to kill radio resistant tumours (fig. 1(d)).

For proton and carbon ions beams with fixed energy most of the dose is deposited in a sharp depth region, called Bragg Peak. In hadrontherapy treatments the flattened dose, a so-called Spread Out Bragg Peak (SOBP), needed to cover all the tumour region, is obtained using beam modifiers or with the overlap of several beams with different energies (fig. 1(c)).

\subsection{Treatment Planning}

In general the most important aspect for radiotherapy is to find for each patient the best modality for the dose distribution, maximising the dose on the tumour while preserving normal healthy tissues. For this task a radiobiological model is needed to predict the cell survival and an optimisation method is required to adjust the delivered biological dose.

Two different beam delivery systems are used in hadrontherapy to conform the dose to the full tumour volume: a passive delivery system, where a fixed energy beam is modelled with beam passive modifiers, and an active delivery system, where the conformation is obtained with ripple filters and by changing direction and energy of a beam steered on the tumour volume.

For the treatment planning in hadrontherapy with active delivery system a complex software, called Treatment Planning System (TPS), is used to optimise the biological dose delivered to the patient and to compute the related physical dose. This has to be done in a general way so that the TPS can be easily adapted for every specific beamline of any hadrontherapy centre. 


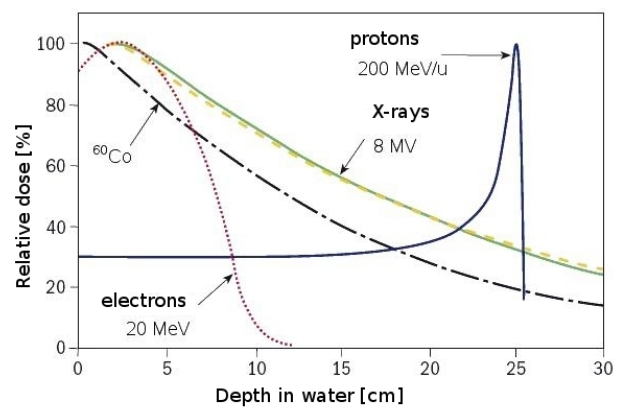

(a) Depth dose distribution for conventional radiation sources and protons.

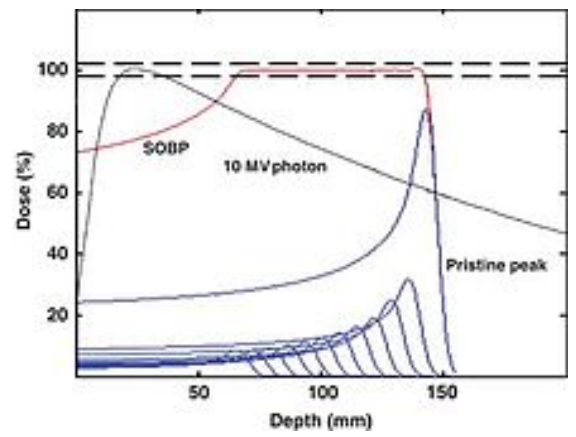

(c) SOBP depth dose distribution for protons compared with proton pristine dose peaks of different energies and X-rays. From [7].

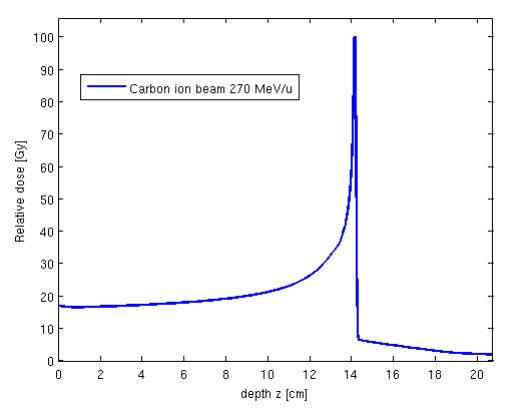

(b) Depth dose distribution for carbon ions.

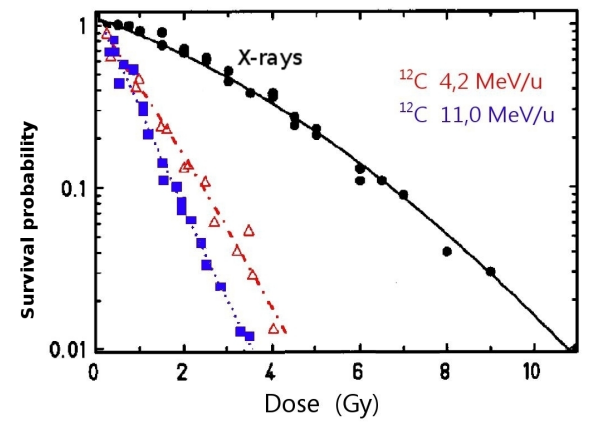

(d) Survival curves for X-rays and carbon ions of different energies.

Figure 1: Dose distribution and survival curves for conventional X-rays compared to protons and carbon ions.

\subsection{Radiobiological modelling}

The optimisation of the biological dose has to include the evaluation of the Relative Biological Effectiveness (RBE) which, for carbon ions, is more complex than for protons. The RBE for a specific radiation type is defined as the quotient of the dose $D_{X-\text { ray }}$ for a reference $\mathrm{X}$-rays radiation and the dose $D_{\text {radiation }}$ for the specific radiation type, both doses producing the same cell survival $S$ :

$$
R B E_{\text {radiation }}=\frac{D_{X-\text { ray }}}{D_{\text {radiation }}} .
$$

For protons the RBE is commonly approximated as constant and equal to 1.1, while for carbon ions it can rise up to about 3 and it is not constant inside the irradiation field. The RBE depends, among other parameters, on the particle type, energy and on the tissue (fig. 1(d)).

The biological dose (also called RBE-weighted dose) for a specific radiation type is defined as:

$$
D_{\text {Biological }}=R B E_{\text {radiation }} \cdot D_{\text {radiation }} \text {. }
$$

The cell survival $S$ is commonly modelled as a function of the physical dose $D$ through the Linear Quadratic (LQ) model:

$$
S(D)=\exp \left(-\alpha \cdot D-\beta \cdot D^{2}\right)
$$


where the two biological parameters, $\alpha$ and $\beta$, in principle depend on many factors, like the type of tissue, the oxygenation level, the physical quality of the radiation, etc.

The parameter $\alpha$ corresponds to the irreparable damage, Double Strand Break (DSB), on the DNA and is dominant for cell lines with low repair capacity and for radiation with high Linear Energy Transfer (LET). The parameter $\beta$ corresponds to reparable damage, Single Strand Break (SSB), on the DNA and is dominant for cell lines with high repair capacity and for radiation with low LET.

Due to the evident difficulties in performing in-vivo experiments, they are estimated on the bases of in-vitro experiments, providing a rough estimate that do not account for cell-cycle or oxygenation variability. Typical parameters for X-rays are $\alpha_{x}=0.18$ and $\beta_{x}=0.028$.

In the case of carbon ions, where so far not many experimental data are available, $\alpha_{c}$ and $\beta_{c}$ are often predicted using a radiobiological model. A candidate for such a model is nowadays the Local Effect Model (LEM) I-IV, developed by the Biophysics group at the Gesellschaft für Schwerionenforschung (GSI), Darmstadt/Germany [8][9][10][11].

\subsection{Present status of proton and carbon ion therapy}

The benefits of carbon ion therapy are not yet clinically proven as the advantages of the proton therapy are, but one expects better results due to a synergetic increase of the RBE along the Bragg Peak [4][5][6].

So far about 67000 patients have been treated with protons since first time in late 1950s in Berkeley, USA, and about 7000 patients with carbon ions since first time in late 1990s in Chiba, Japan [13]. Nowadays the number of operating proton therapy centres is about 35 and it is steadily growing, while the operating carbon ion centres are only 5 with other 5 currently under construction:

- NIRS-HIMAC, Chiba (since 1994), HIBMC, Hyogo (since 2002) and GHMC, Gunma (since 2010) in Japan.

- HIT, Heidelberg (since 2009), PTC, Marburg (planned for 2011) and NRoCK, Kiel (planned for 2012) in Germany.

GSI, a pilot project in which almost 400 patients have been treated between 1997 and 2007.

- IMPCAS, Langzhou (since 2010) and HITFil, Langzhou (planned for 2013) in China.

- CNAO, Pavia (planned for 2011) in Italy.

- Med-AUSTRON, Wien (planned for 2014) in Austria.

\section{The INFN TPS project}

The scope of the INFN TPS project is to implement a fully functional TPS for therapy systems using protons and carbon ions with active dose delivery [15].

It is a multi-disciplinary project involving many sections of the Istituto Nazionale di Fisica Nucleare (INFN) and belonging to different research areas, as for example nuclear physics, radiobiology, optimization and Monte Carlo methods (using Fluka [14]) and beam dose verification with Positron Emission Tomography (PET) (fig. 2). 
Among others it is planned to measure the fragmentation cross-sections of the ion fragmentation products at Laboratori Nazionali del Sud (LNS), Catania/Italy, and within the experiment FIRST (Fragmentation of Ions Relevant for Space and Therapy), which has been approved for data taking at the GSI synchrotron in summer 2011.

Furthermore, since 2009 the TPS is part of an industrial cooperation agreement between the INFN and the Belgian Ion Beam Applications (IBA) company. Other two Belgian partners, Cenaero and Qspin, are part of the collaboration and will work respectively on the optimisation methods and the software quality assurance.

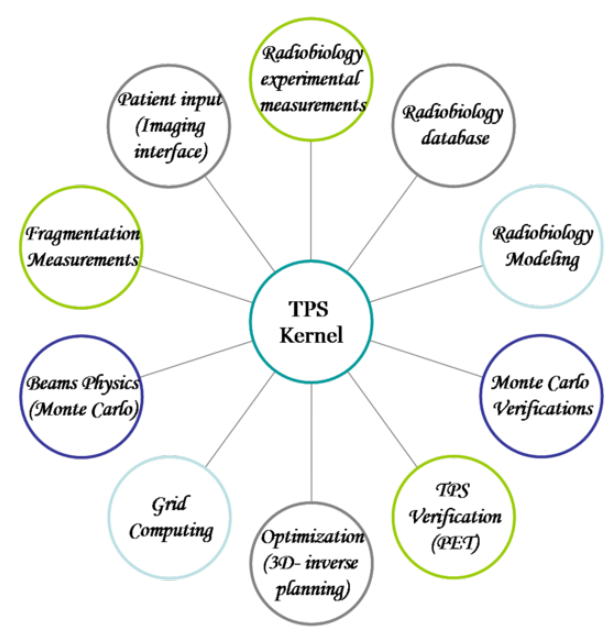

Figure 2: The INFN TPS research areas.

\section{Biological dose optimisation}

In a treatment with an active delivery system, the dose to the tumour is produced by the superposition of multiple quasi-parallel beams, hereafter called beamlets, each of them characterised by a set of parameters, namely its energy $E$, direction $\vec{d}$ and fluence $\phi$.

The aim of the TPS is to determine the parameters $(E, \vec{d}, \phi)$ for each beamlet which optimise the actual delivered biological dose with respect to the medical prescription, translated into a mathematical formulation in terms of a cost function. In general the formulation of the cost function is of great importance to obtain good results for the biological dose (and fluence) distribution.

The beamlet directions $\vec{d}$ depend on the geometry of the tumour and the direction steps $\Delta \vec{d}$ are chosen in such a way that a flat lateral dose distribution is obtained with the overlapping of the beamlets. A common rule is that the distance between two adjacent beamlets is equal to $\frac{1}{3}$ of the transverse beam Full Width Half Maximum (FWHM). Similar arguments apply to the choice of the energy steps $\Delta E$, considering the width of the Bragg Peak as enlarged by passive elements placed along the beam path.

The only remaining free parameters are the fluences $\phi$, which have to be determined with an optimisation method. Before discussing how the optimisation is performed, the radiobiological effect of the overlap of different beamlets has to be discussed.

In contrast to the traditional radiotherapy, the RBE of charged particles is not constant, and this renders the optimisation more complex. Furthermore the determination of the RBE has to take into account the complexity of the energy deposition processes. Indeed the deposition is the result of a mixed field, because in every point of the patient the dose is delivered by particles of different species and energies, due to fragmentation and energy degrading of the beamlet particles; each of these particles has a different capability of cell killing. 
It has been observed [12] that a good approximation for the average radiobiological beamlet effectiveness can be achieved with the following formulation:

$$
\alpha_{i}(q)=\frac{\sum_{l=1}^{L} \alpha_{l, i}(q) \cdot D_{l, i}(q)}{\sum_{l=1}^{L} D_{l, i}(q)}, \quad \beta_{i}(q)=\left(\frac{\sum_{l=1}^{L} \sqrt{\beta_{l, i}(q)} \cdot D_{l, i}(q)}{\sum_{l=1}^{N} D_{l, i}(q)}\right)^{2}, \quad D_{i}(q)=\sum_{l=1}^{L} D_{l, i}(q)
$$

The sums extend to the radiobiological parameters $\alpha_{l, i}(q), \beta_{l, i}(q)$ and to the dose deposition $D_{l, i}(q)$ of the different particles arising from a single beamlet $i$ (also called elementary field) $i$ at a certain point $q=(x, y, z) \in \mathbb{R}^{3}$.

Taking into account the average values for each of the elementary fields $i$, the radiobological effectiveness for the whole set of the beamlets is derived using the following parameters:

$$
\alpha(q)=\frac{\sum_{i=1}^{N} \alpha_{i}(q) \cdot D_{i}(q)}{\sum_{i=1}^{N} D_{i}(q)}, \quad \beta(q)=\left(\frac{\sum_{i=1}^{N} \sqrt{\beta_{i}(q)} \cdot D_{i}(q)}{\sum_{i=1}^{N} D_{i}(q)}\right)^{2}, \quad D(q)=\sum_{i=1}^{N} D_{i}(q),
$$

where $\alpha_{i}(q), \beta_{i}(q)$ and $D_{i}(q)$ are respectively the biological parameters and the physical dose for each elementary field $i$. Using this prescription it is possible to calculate the parameters $\alpha_{c}$ and $\beta_{c}$ for the mixed field encountered in therapies with carbon ion beams.

The dose optimisation problem consists to find the minimum of the biological cost function with respect to the number of primary particles $M$, or respectively the fluences $\phi_{i}$ for each of the $i=1, \ldots, N$ beamlets.

An example of the cost function is given by the following equation [20]:

$$
\chi^{2}(M)=\sum_{q \in \text { tuтоит }} \frac{\left(D_{p}(q)-D_{a}(q, M)\right)^{2}}{\Delta D_{p}^{2}(q)}+\sum_{q \in O A R} \frac{\left(D_{p}(q)-D_{a}(q, M)\right)^{2}}{\Delta D_{p}^{2}(q)} \cdot \theta\left(D_{p}(q)-D_{a}(q, M)\right) .
$$

Where $D_{p}(q)$ is the prescribed biological dose for each volume at the location $q$ and $D_{a}(q, M)$ represents the actual biological dose generated by the beam configuration $M$ at this location:

$$
D_{a}(q, M)=\sum_{i=1}^{N} R B E_{i}\left(q, \phi_{i}\right) \cdot D_{i}\left(q, \phi_{i}\right)
$$

The biological dose tolerance $\Delta D_{p}(q)$ is defined as

$$
\Delta D_{p}(q)=f_{v} \cdot \frac{D_{p}(q)}{w_{v}(q)}
$$

where the parameter $f_{v}$ is the allowed average deviation of the actual biological dose (typically chosen as $\left.f_{v}=0.025\right)$ and $w_{v}(q) \geq 0$ are the weights imposed to each Volume Of Interest (VOI) $v$, the tumour or the OAR, chosen on the bases of the clinical experience.

For the cost function in the example, the OAR terms are modelled with a penalty function $\theta$, different from zero only when $D_{a}$ exceeds $D_{p}$. 
The prescription $D_{p}$ has been assigned on the bases of clinical experience with reference to $\mathrm{X}$-ray, the related biological effect can be easily derived in terms of the cell survival (see equation $1.3)$ :

$$
S_{x}\left(D_{p}\right)=\exp \left(-\alpha_{x} \cdot D_{p}-\beta_{x} \cdot D_{p}^{2}\right) .
$$

The cell survival $S_{x}$ is in fact the end-point that has to be kept equal to the cell survival $S_{c}$ for carbon ions, which has a RBE different from one, as assumed for X-rays. This request leads to the following equation (with $D$ being the physical dose for the whole set of the carbon ion beamlets):

$$
S_{x}\left(D_{p}\right)=S_{c}(D)=\exp \left(-\alpha_{c} \cdot D-\beta_{c} \cdot D^{2}\right),
$$

or in other terms (see equation 1.2)

$$
D(q, M)=\frac{D_{p}(q)}{R B E(q, M)} .
$$

The problem is complicated by the fact that the RBE for carbon ions changes along the threedimensional beamlet propagation and by the non-linearity of the problem.

Furthermore, it has to be to remarked that the robustness of the treatment plan for charged particle beams delivered with an active delivery system is more vulnerable with respect to a passive system or standard X-ray therapy, as the fluctuations of many beamlets, contemporarily involved, have to be taken into account. This issue has to be dealt with by including extra-terms for the fluence distribution in the cost function.

For all the reasons explained before, it can be concluded that the optimisation is a complex process. This is furtherly amplified by the great number of variables, which is of the order of $10^{5}$. An advanced optimisation method is necessary, considering that the time to complete a treatment planning is an important parameter. In general a gradient based method is used instead derivative free methods like for example simplex methods or full Monte Carlo search algorithms [20]. In the past there have been several developments regarding general methods to obtain a minimum of a function of thousands of free parameters, like the well-known Steepest Descent (SD), Conjugate Gradient $(C G)$ and Quasi Newton $(Q N)$ methods; the last two mentioned ones being implemented as standard algorithms in many TPS. To reduce hereby as much as possible the number of iterations, it is necessary to determine the first derivatives of the cost function with respect to the free parameters.

Besides the optimisation algorithm it is of importance the choice of the starting vector, that is the set of values for the free parameters to start the minimisation. The speed of the minimisation is highly increased if the starting values assigned to the fluences are close to the final ones. The most commonly used basic approaches are two: optimising only the physical dose and including the OAR or optimising already the biological dose but excluding the OAR [20].

\subsection{TPS prototype conditions}

A TPS prototype has been implemented in Matlab [21] to study the performances of different optimisation techniques.

The input to the prototype were a water phantom description and a definition of the VOI with the 
respective prescription. This patient case was then mapped to a pre-computed beam library, derived from the geant 4 [22] simulation of the fragmentation processes produced by carbon ions in water. The energy deposition and the description of particle spectra produced by the beamlet as a function of depth and distance from the beam axis have been derived by tracing the beamlet within the whole volume. In addition the appropriate biological parameters $\alpha$ and $\beta$, needed to perform the optimisation of the biological dose, were used as input.

To mimic a realistic beamlet shape the design parameters of the Centro Nazionale di Adroterapia Oncologica (CNAO), Pavia/Italy, beamline have been used. The beamlet shape was simulated having a $F W H M \sim 14 \mathrm{~mm}$ in both transverse directions. The spread of the Bragg peak, which depends on the beamlet energy, has a width that spans from $F W H M=3.7 \mathrm{~mm}$ for $60 \mathrm{MeV} / \mathrm{u}$ to $F W H M=12 \mathrm{~mm}$ for $490 \mathrm{MeV} / \mathrm{u}$. As already stated before, the spot steps used should be $\frac{1}{3}$ of the beamlet FWHM, where the relation between the beamlet FWHM and the beamlet sigma $\sigma$ is given through

$$
F W H M=2 \cdot \sqrt{2 \cdot \ln (2)} \cdot \sigma
$$

For each point $q$ the dose deposited due to a single beamlet, $D_{i}(q)$, was thus evaluated for unit fluence. To account for the radiobiological effects, $\alpha_{c}$ and $\beta_{c}$ were evaluated in 3-dimensions with the $L E M$.

\subsubsection{Optimization set-up for a basic treatment example}

A small rectangular phantom corresponding to $31 \times 31 \times 101$ cubic voxels of $2 \mathrm{~mm}$ size was used to test the performance of different local optimisation methods and their start vector dependence. The treatment volume was made of $6 \times 6 \times 14$ voxels located roughly in the center of the phantom. The points where the dose calculation was computed, the so-called calculation grid, were placed in the center of each voxel.

The energies used for this treatment example were $270 \mathrm{MeV} / \mathrm{u}$ (with a Bragg Peak of $F W H M=$ $15 \mathrm{~mm}$ and $\left.\sigma_{z}=6.37 \mathrm{~mm}\right), 240 \mathrm{MeV} / \mathrm{u}\left(F W H M=13 \mathrm{~mm}, \sigma_{z}=5.52 \mathrm{~mm}\right)$ and $200 \mathrm{MeV} / \mathrm{u}$ $\left(F W H M=11 \mathrm{~mm}, \sigma_{z}=4.67 \mathrm{~mm}\right)$. For the transverse direction the fixed $\sigma_{x, y}=6.00 \mathrm{~mm}$ corresponds to a FWHM of $14.13 \mathrm{~mm}$. According to the beamlet FWHM in the transverse plane and to the spread of the Bragg Peak, the beam spot positions were spaced of about $4.71 \mathrm{~mm}$ in $x$ and $y$ and $5.10 \mathrm{~mm}$ along $z$, producing the spot grid. The resulting number of beamlets was thus 175 .

The dose prescription was set to $2 \mathrm{~Gy}$ for the tumour and to $0 \mathrm{~Gy}$ for the OAR, with a weight on the tumour $w_{\text {tumour }}=1$. Furthermore the weight on the OAR was changed between $w_{O A R}=0$ and $w_{O A R}=0.1$.

The fluence start vector was set either constant to $f_{D}$ (with $f_{D}$ being a normalisation factor depending on the prescribed dose $D_{p}$ ) or to the pre-optimised fluence vector, here obtained by the previous optimisation excluding the OAR.

The equation 3.3, following a traditional approach in which the biological dose is optimised directly, was used as cost function. In this case $D_{p}=0$ for all OAR, the penalty function was set to a constant $\theta=1$ and the dose tolerance to $\Delta D_{p}(q)^{-1}=w_{v}(q)$. This leads to the following simplified 
cost function

$$
\chi^{2}(M)=\sum_{q \in \text { tumour }}\left\{w_{\text {tumour }} \cdot\left(D_{p}(q)-D_{a}(q, M)\right)\right\}^{2}+\sum_{q \in O A R}\left\{w_{O A R} \cdot\left(D_{p}(q)-D_{a}(q, M)\right)\right\}^{2} .
$$

The possibility of having an alternative cost function based on the lethal events $L_{x}$ and $L_{c}$ constructed using equations 3.6 and 3.7 has been also explored. The number of lethal events is derived directly from the cell survival using the relationship:

$$
L_{c}(D)=-\ln \left(S_{c}(D)\right)=\alpha_{c} \cdot D+\beta_{c} \cdot D^{2}
$$

with the biological parameters $\alpha_{c}, \beta_{c}$ and the physical dose $D$ as derived from equation 3.2. All together this leads to a cost function of the form

$\chi^{2}(M)=\sum_{q \in \text { tumour }}\left\{w_{\text {tumour }} \cdot\left(L_{x}\left(D_{p}(q)\right)-L_{c}(D(q, M))\right)\right\}^{2}+\sum_{q \in O A R}\left\{w_{O A R} \cdot\left(L_{x}\left(D_{p}(q)\right)-L_{c}(D(q, M))\right)\right\}^{2}$.

With this cost function it is expected that the optimisation problem needs less iterations to converge and that the fluence distribution leads to a more robust solution.

\subsection{Results and outlook}

- Fig. 3(a) shows the prescription for the biological dose released in the phantom, while fig. 3(b) shows the biological dose distribution for the initial forward planning, in which constant normalised fluences are used.

The results from the optimisation based on the cost function constructed by the biological dose differences of equation 3.10 and using the CG method are shown in fig. 3(c), 3(e), 4(a) and 4(c).

Assuming no OAR (the corresponding weight was set to $w_{O A R}=0$ ) the biological dose distribution obtained with the optimisation and shown in fig. 3(c) matches the dose prescription. The $\chi^{2}$ of the optimised biological dose $D_{a}$ with respect to the prescribed biological dose $D_{p}$ refers hereby to equation 3.10. The resulting optimised fluences from this optimisation case are reported in fig. 3(e).

By adding the request on the OAR (by setting $w_{O A R}=0.1$ ) the results shown in fig. 4(a) are obtained. The speed improvement due to an appropriate start vector for this case is shown in fig. 4(c). The dose distribution is as good as in fig. 4(a), using the constant normalised fluence start vector, but needs much less iterations. The same is valid for the fluence distribution.

The same configurations were studied using the cost function constructed by the lethal events differences of the actual physical dose, produced by the carbon ion beamlets, and the prescribed biological dose, as from equation 3.12. The results are shown in fig. 3(d), 3(f), 4(b) and $4(\mathrm{~d})$. The $\chi^{2}$ refers again to equation 3.10, using the biological dose $D_{a}$ obtained with the optimisation and compared to the prescribed biological dose $D_{p}$. The biological dose distributions are very similar to the ones obtained with the conventional cost function, while 


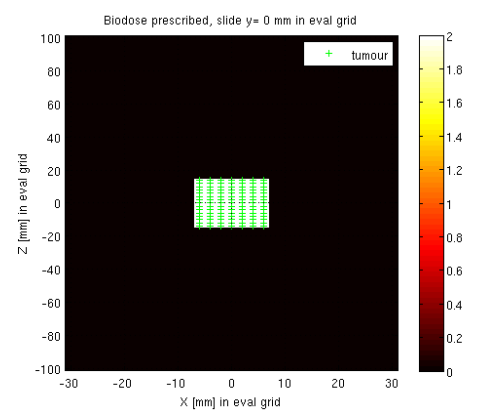

(a) Biological dose prescription (Gy).

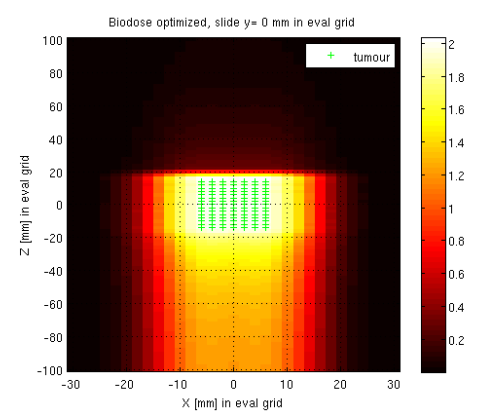

(c) Optimised biological dose (from biological dose cost function), $\chi^{2}=0.2210$ with 13 iterations.

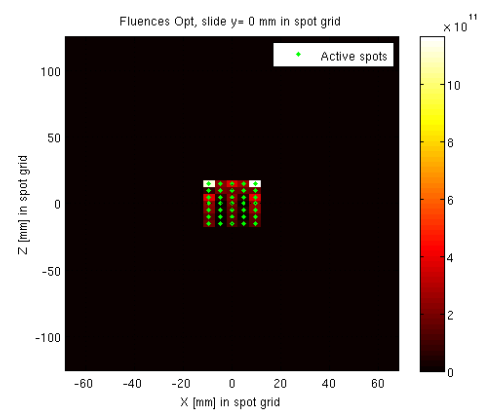

(e) Optimised fluences (from biological dose cost function), $\chi^{2}=0.2210$ with 13 iterations.



(b) Biological dose (Gy) obtained from constant normalised fluence starting vector $f_{D}$.

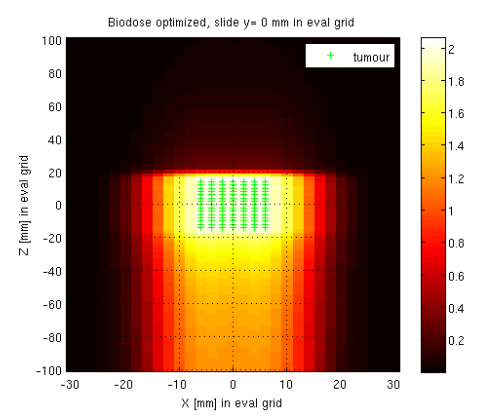

(d) Optimised biological dose (from lethal events cost function), $\chi^{2}=0.2593$ with 8 iterations.

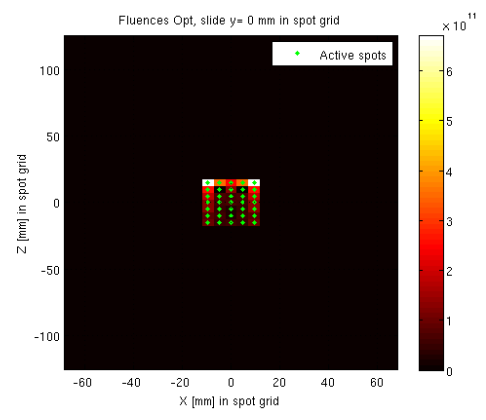

(f) Optimised fluences (from lethal events cost function), $\chi^{2}=0.2593$ with 8 iterations.

Figure 3: Biological dose prescription and dose optimisation (CG method with constant normalised starting vector) results excluding the OAR.

the number of iterations have decreased in two out of three cases and the fluence distributions show less fluctuations.

- Some care has to be taken in the choice of the optimisation starting vector for the initial fluence distribution. As suggested by [20] the pre-optimised fluences obtained by optimising the biological dose without constraints on the OAR $\left(w_{O A R}=0\right)$ were used. Another possibility could be to obtain the start vector from pre-optimisation through modelling only the physical dose and including the OAR. 


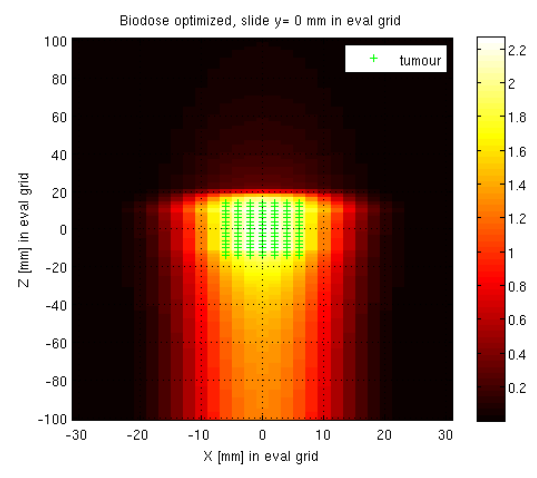

(a) Optimised biological dose (from biological dose cost function), $\chi^{2}=121.7765$ with 38 iterations.

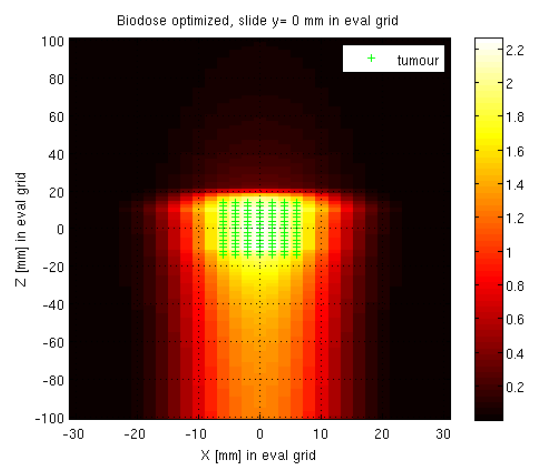

(c) Optimised biological dose (from biological dose cost function, using starting vector obtained from previous optimisation without OAR), $\chi^{2}=121.7769$ with 25 iterations.

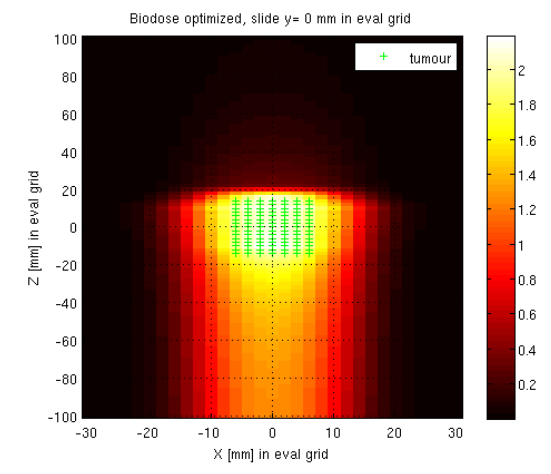

(b) Optimised biological dose (from lethal events cost function), $\chi^{2}=125.4754$ with 39 iterations.



(d) Optimised biological dose (from lethal events cost function, using starting vector obtained from previous optimisation without OAR), $\chi^{2}=126.7488$ with 15 iterations.

Figure 4: Biological dose optimisation (CG method with constant normalised starting vector and starting vector from previous optimisation without OAR) results including the OAR.

The optimisation can lead to results that are optimised but the fluence distribution suffers of large fluctuations (no treatment plan robustness). The effect has to be avoided with further constraints on the fluence distribution.

It is planned to implement and test other optimisation methods, as for example the local first order SD and second order QN method [24][23], as both are, beside CG, the referred standard methods in dose optimisation. For their application the first and second derivatives of the cost function must be computed analytically or numerically.

A more realistic set-up will be implemented to test more precisely the performance of different optimisation algorithms and cost functions.

\section{Beam modelling for the TPS kernel}

For a given hadrontherapy centre and beamline the physical dose distribution $D(q, E)$ at a point $q=(x, y, z) \in \mathbb{R}^{3}$, deriving from a beamlet of energy $E$ (in the following called nozzle beam), can be measured with relatively standard detectors or simulated by Monte Carlo methods, while the 
radiobiological effect, parameterised in terms of $\alpha(q, E)$ and $\beta(q, E)$, can be determined only by time and resources consuming cell survival experiments or biological simulations.

The possibility of deriving these radiobiological parameters by superimposing the effect of multiple dimensionless monoenergetic beams (so-called pencil beams) of an energy $E^{*}$ close to the nominal one $E$, weighted by a Beamline Transfer Function $(B T F) f(q, E)$, has been investigated.

Assuming that each of the $i=1, \ldots, N$ pencil beams is characterised by $\alpha_{0}\left(q, E_{i}^{*}\right)$ and $\beta_{0}\left(q, E_{i}^{*}\right)$ and by the deposited dose $D_{0}\left(q, E_{i}^{*}\right)$, it is expected that the superposition with appropriate weights $w\left(q, E_{i}^{*}\right)$ of multiple pencil beams describes $\alpha(q, E)$ and $\beta(q, E)$.

In the present study, the BTF was derived from the comparison between the physical dose distributions $D(q, E)$ and $D_{0}\left(q, E_{i}^{*}\right)$ as

$$
D(q, E)=\sum_{i=1}^{N} D_{0}\left(q, E_{i}^{*}\right) \cdot w\left(q, E_{i}^{*}\right) .
$$

To derive the biological parameters $\alpha(q, E)$ and $\beta(q, E)$, equation 3.2 was applied to the case of a superposition of multiple pencil beams. The formulation leads to:

$\alpha(q, E)=\frac{\sum_{i=1}^{N}\left\{\alpha_{0}\left(q, E_{i}^{*}\right) \cdot D_{0}\left(q, E_{i}^{*}\right)\right\} \cdot w\left(q, E_{i}^{*}\right)}{\sum_{i=1}^{N} D_{0}\left(q, E_{i}^{*}\right) \cdot w\left(q, E_{i}^{*}\right)}, \quad \beta(q, E)=\left(\frac{\sum_{i=1}^{N}\left\{\sqrt{\beta_{0}\left(q, E_{i}^{*}\right)} \cdot D_{0}\left(q, E_{i}^{*}\right)\right\} \cdot w\left(q, E_{i}^{*}\right)}{\sum_{i=1}^{N} D_{0}\left(q, E_{i}^{*}\right) \cdot w\left(q, E_{i}^{*}\right)}\right)^{-2\}}$.

The 3-dimensional BTF $f(q, E)$ can be factorised in a longitudinal part $f(z, E)$ along the beamline and a transversal part $f(x, y, E)$.

\subsection{Ripple filter example for a carbon ion beam}

In the following an application considering only the longitudinal part of the BTF for a special case represented by a ripple filter, as sketched in uppermost of fig. 5(a), is presented. The ripple filter is a common device used to spread the single beam Bragg Peaks in order to obtain a flat dose distribution, when constructing the SOBP.

Fig. 5(b) shows the the longitudinal physical dose distribution of the single pencil beam and of the same beam which has passed through the ripple filter (that means the nozzle beam), for carbon ions of $\mathrm{E}=270 \mathrm{MeV} / \mathrm{u}$. The ripple filter affects the beam in a way that can be described analytically, in other terms the analytical form $\tilde{f}(z, E)$ of the BTF is known (lower part of fig. 5(a)).

The connection between the pencil beam dose $D_{0}\left(z, E^{*}\right)$ and the nozzle beam dose $D(z, E)$ is given through the $\operatorname{BTF} f(z, E)$, which has to be determined from the physical dose distributions and to be compared with its analytical form $\tilde{f}(z, E)$. Due to noise or to the methods used to determine $f(z, E)$, it can be also different from its analytical form $\tilde{f}(z, E)$.

Two methods have been implemented in Matlab and applied to determine the BTF. The first one was a classical de-convolution approach, using the Discrete Fourier Transform (DFT) $\mathscr{F}$ and the inverse DFT $\mathscr{F}^{-1}$ to find the BTF $f(z, E)$ as a linear filter :

$$
D(z, E)=D_{0}\left(z, E^{*}\right) * f(z, E) \quad \Leftrightarrow f(z, E)=\mathscr{F}^{-1}\left(\frac{\mathscr{F}(D(z, E))}{\mathscr{F}\left(D_{0}(z, E)\right)}\right) .
$$



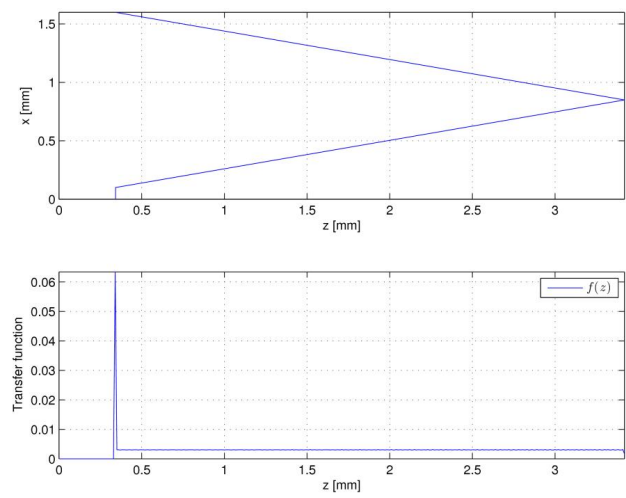

(a) Ripple filter and resulting analytical transfer function $\tilde{f}(z, E)$ (shown inverse for convolution).

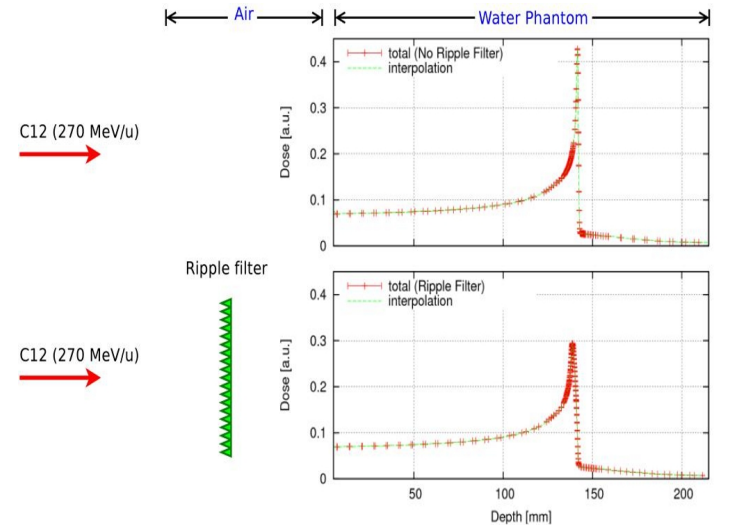

(b) Carbon ion pencil and nozzle beam modelled with a ripple filter.

Figure 5: Ripple filter $270 \mathrm{MeV/u}$ carbon ions example.

This approach determines a BTF $f(z, E)$, which reproduces qualitatively the analytical transfer function $\tilde{f}(z, E)$ and which leads to a good agreement between the reconstructed and the original nozzle beam, both for the physical and for the biological simulations. Nevertheless, the method suffers from noise and furthermore it is not strictly leading to a positive solution over all the range, as it has to be due to the physical requirements.

The second approach was an optimisation method for a superposition of $i=1, \ldots, N$ pencil beams of energies $E_{i}^{*}$, weighted with the unknown weight function $\vec{w}\left(z, E^{*}\right):=\left(w\left(z, E_{1}^{*}\right), \ldots, w\left(z, E_{N}^{*}\right)\right)$ as follows:

$$
D(z, E) \approx \sum_{i=1}^{N} w\left(z, E_{i}^{*}\right) \cdot D_{0}\left(z, E_{i}^{*}\right), \quad E_{i}^{*} \sim E .
$$

The different dose curves $D_{0}\left(z, E_{i}^{*}\right)$ were approximated through only one dose curve $D_{0}\left(z, E^{*}\right) \approx$ $D_{0}\left(z, E_{i}\right)$ of an average energy $E^{*}=270 \mathrm{MeV} / \mathrm{u}$, shifted M-times with certain regular steps $\Delta z_{k}$ within a certain maximum range so that for $\tilde{z}=\left(z-\Delta z_{1}, \ldots, z-\Delta z_{M}\right)$ we obtained

$$
D(z, E) \approx \sum_{k=1}^{M} w_{k} \cdot D_{0}\left(\tilde{z}_{k}, E^{*}\right), \quad w_{k}:=w\left(\tilde{z}_{k}, E^{*}\right)
$$

with the new weight function $\vec{w}\left(\tilde{z}, E^{*}\right):=\left(w_{1}, \ldots, w_{M}\right)$. The number of pencil beams needed to describe the nozzle beam is in general rather limited, that means less than $10^{2}$, and the optimisation problem is linear. Thus the optimisation procedure, using for example the CG method, is relatively straightforward.

The described weight optimisation method leads to a BTF $f(z, E)=\vec{w}\left(\tilde{z}, E^{*}\right)$ which reproduces qualitatively the analytical transfer function $\tilde{f}(z, E)$ and which shows good results for the reconstructed physical and biological simulations already with few pencil beams spaced by $1 \mathrm{~mm}$. In general the spacing should be adapted to the particle type and energy, choosing more or less $\frac{1}{3}$ of the pencil beam FWHM. This weight optimisation method has the advantages that the results are almost not affected by noise and that the weights are always positive. 


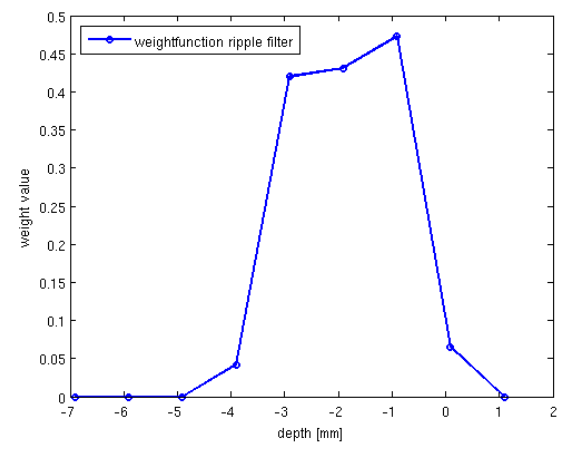

(a) Weight function obtained by optimisation with $1 \mathrm{~mm}$ steps and 9 shifted beams performed on $50 \%$ relative dose region.

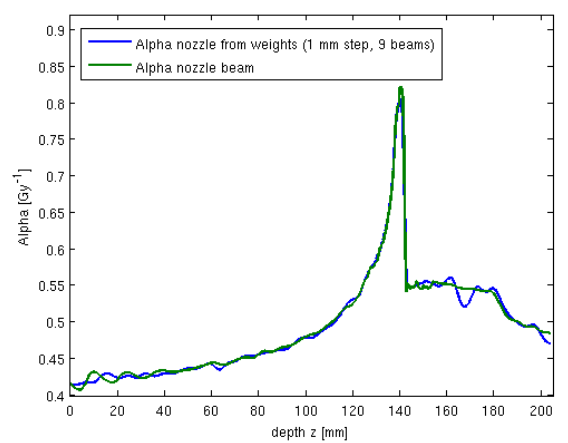

(c) $\alpha$ obtained by optimisation with $1 \mathrm{~mm}$ steps and 9 shifted beams compared to the original nozzle beam LEM simulation.



(b) Physical dose obtained by optimisation with 1 mm steps and 9 shifted beams compared to the original nozzle beam MC simulation.

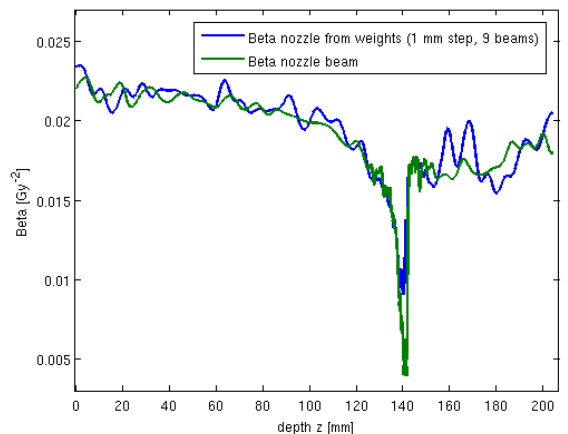

(d) $\beta$ obtained by optimisation with $1 \mathrm{~mm}$ steps and 9 shifted beams compared to the original nozzle beam LEM simulation.

Figure 6: BTF weights and reconstructed nozzle beam results compared with the original nozzle simulation.

\subsection{Results and outlook}

- In the following only the results obtained with the weight optimisation method applied on the ripple filter example are discussed.

Fig. 6(a) shows the distribution of the weights for 9 beams with a step size of $1 \mathrm{~mm}$, while fig. 6(b) shows the reconstructed nozzle beam dose curve compared to the original nozzle beam simulation, demonstrating a good agreement. Fig 6(c) and 6(d) represent the obtained reconstructed nozzle beam radiobiological parameters $\alpha$ and $\beta$ compared to the original nozzle beam simulations, showing also here a good agreement.

- It is planned to extend the BTF method to a clinical three-dimensional case and to check the results with respect to formal quality aspects for proton and carbon ion therapies.

\section{Conclusions}

The INFN TPS project has been presented, focusing on two particular tasks: the biological dose optimisation and the beam modelling. 
A first implementation of the biological dose optimisation with a basic cost function and a CG optimisation method has been successfully tested on a restricted volume. In the future more advanced optimization and start vector methods as well as cost functions and the inclusion of their derivatives will be developed and applied on realistic treatment volumes.

Considering the beam modelling for the physical and biological data, a first implementation and test of the BTF method has been presented, focusing only on the longitudinal part of the BTF. The method has been checked on different settings, even though here only the ripple filter example was discussed, and the results are quite promising.

The present work describes only a small part of all the ongoing research activities within the INFN TPS project and in hadrontherapy in general. Interesting new results are to be expected in this field, leading to applications to proton and specially to the much newer carbon ion therapy.

\section{References}

[1] Website of the ECO (European Cancer Observatory): http://eu-cancer.iarc.fr

[2] Website of the EPHA (European Public Health Alliance): http://epha.org

[3] Website of the WHO (World Health Organization): http://www.who.int

[4] U. Amaldi G. Kraft: Radiotherapy with beams of carbon ions, Rep. Prog. Phys. 68, 1861-1882 (2005)

[5] G. Kraft and S. D. Kraft: Research needed for improving heavy-ion therapy, New Journal of physics 11, (2009)

[6] D. Schardt and T. Elsässer: Heavy-ion tumor therapy: Physical and radiobiological benefits, Reviews of modern physics 82, 383-425 (2010)

[7] W. P. Levin, H. Kooy, J. S. Loeffler and T. F. DeLaney: Proton beam therapy, British Journal of Cancer 93, 849-854 (2005)

[8] M. Scholz and G. Kraft: Track structure and the calculation of biological effects of heavy charged particles, Adv. space Res. 18, 5-14 (1996)

[9] T. Elsässer and M. Scholz: Cluster effects within the local effect model, Radiat Res. 167, 319-329 (2007)

[10] T. Elsässer, M. Krämer and M. Scholz: Accuracy of the local effect model for the prediction of biologic effects of carbon ion beams in vitro and in vivo, Int. J. Radiat. Oncol. Bio. Phys. 71, 866-872 (2008)

[11] T. Elsässer et al: Quantification of the relative biological effectiveness for ion beam radiotherapy: Direct experimental comparison of proton and carbon ion beams and a novel approach for treament planning, Int. J. Radiat. Oncol. Bio. Phys. 78, 1177-1183 (2010)

[12] M. Zaider and H. H. Rossi: The Synergistic Effects of different Radiations, Radiation Research 83, 732-739 (1980)

[13] Website of the Particle Therapy Co-Operative Group (PTCOG): http://ptcog.web.psi.ch

[14] Official fluka website: http://www.fluka.org/fluka.php

[15] Website of the INFN TPS project: http://totlxl.to.infn.it/mediawiki/index.php/Main_Page 
[16] M. Krämer et al: Treatment planning for heavy-ion therapy: physical beam model and optimization, Phys. Med. Biol. 45, 3299-3317 (2000)

[17] M. Krämer and M. Scholz: Treatment planning for heavy-ion therapy: calculation and optimization of biologically effective dose, Phys. Med. Biol. 45, 1101-1116 (2001)

[18] M. Krämer: Treatment planning for heavy-ion therapy: biological optimization of multiple beam ports, J. Radiat. Res. 42, 39-46 (2001)

[19] O. Jäkel et al: Treatment planning for heavy-ion therapy: clinical implementation and application, Phys. Med. Biol. 46/4, 3319-3330 (2000)

[20] A. Gemmel et al: Biological dose optimization with multiple ion fields, Phys. Med. Biol. 53, 6991-7012 (2008)

[21] Official matlab website: http://www.mathworks.com

[22] Official geant4 website: http://geant4.cern.ch

[23] F. Albertini et al: Planning and optimizing treatment plans for actively scanned proton therapy, Proc. Biomedical Mathematics: Promising directions in imaging, therapy planning and inverse problems, 1-18 (2009)

[24] F. Albertini et al: The influence of the optimization starting conditions on the robustness of intensity-modulated proton therapy plans, Phys. Med. Biol. 55, 2863-2878 (2010) 